\title{
A Periodic DFT Study of the Synergistic Mechanisms between Extraframework Aluminum Species and Bronsted Acid Sites in HY Zeolites
}

\author{
Jian Zheng ${ }^{\dagger,}$, Yucai Qin*, Qiang $\mathrm{Li}^{\ddagger}$, Li Zhang ${ }^{\S, \|}$, \\ Xionghou Gao*, ${ }^{*,}$ Lijuan Song*,†,
}

${ }^{\dagger}$ College of Chemistry \& Chemical Engineering, China University of Petroleum (East China),

Qingdao 266555, Shandong, P. R. China

${ }^{\ddagger}$ Key Laboratory of Petrochemical Catalytic Science and Technology, Liaoning Province,

Liaoning Shihua University, Fushun 113001, Liaoning, P. R. China

${ }^{\S}$ Lanzhou Petrochemical Research Center, Petrochemical Research Institute,

PetroChina Company Limited, Lanzhou 730060, P. R. China

"Department of Chemistry, Lanzhou University, Lanzhou 730000, China.

*To whom correspondence should be addressed: 1song56@263.net (L.S.). 


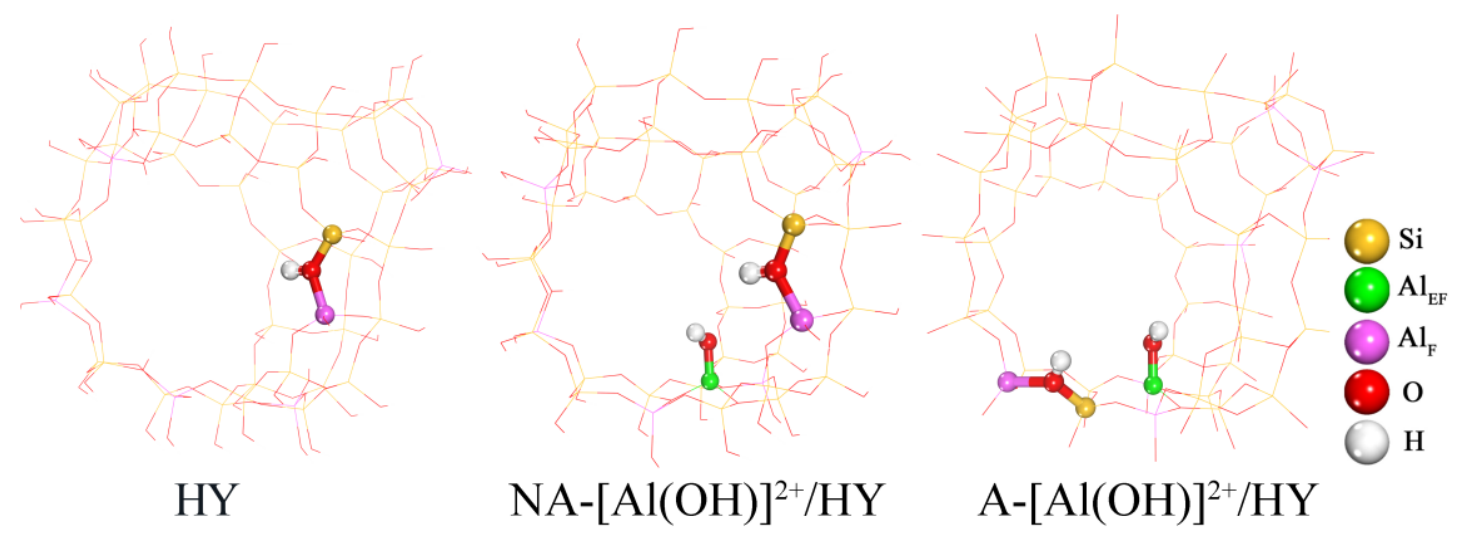

Figure S1 Optimized structures of HY, NA-[Al(OH) $]^{2+} / \mathrm{HY}$ and $\mathrm{A}-[\mathrm{Al}(\mathrm{OH})]^{2+} / \mathrm{HY}$ zeolite cluster models. 
(a)

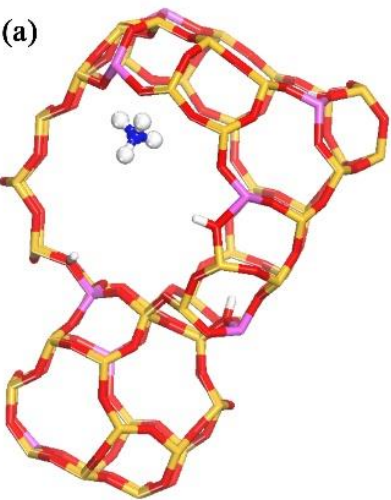

$\mathrm{NH}_{3}-\mathrm{HY}$

(d)

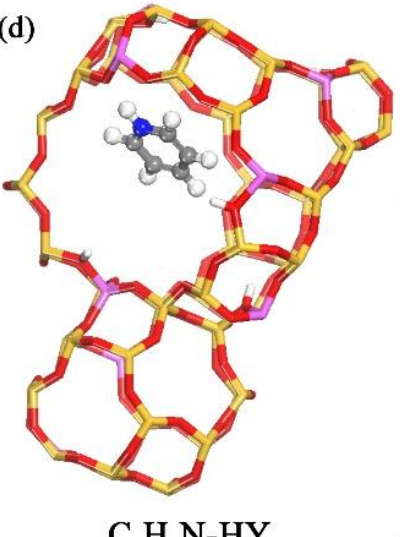

$\mathrm{C}_{5} \mathrm{H}_{5} \mathrm{~N}-\mathrm{HY}$

(g)

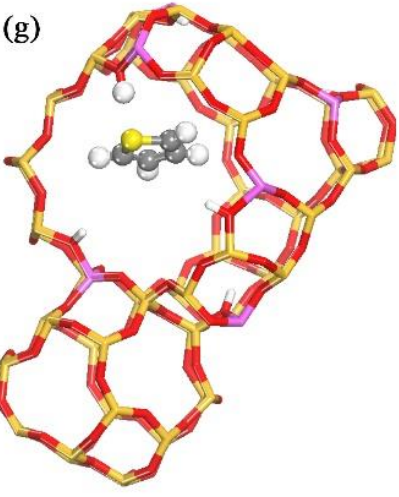

$\mathrm{C}_{4} \mathrm{H}_{4} \mathrm{~S}-\mathrm{HY}$ (b)

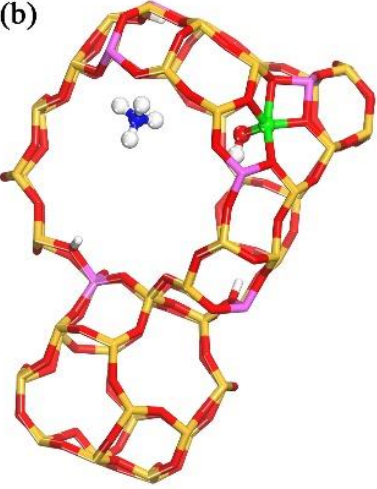

$\mathrm{NH}_{3}-\mathrm{NA}-[\mathrm{Al}(\mathrm{OH})]^{2+} / \mathrm{HY}$

(e)

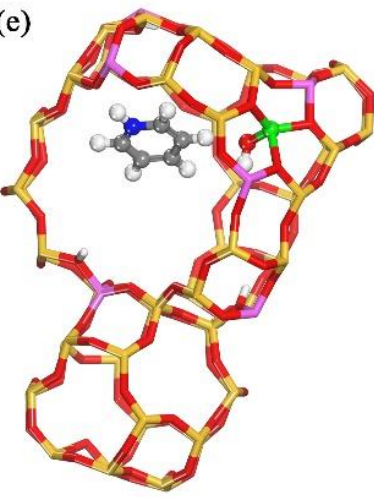

$\mathrm{C}_{5} \mathrm{H}_{5} \mathrm{~N}-\mathrm{NA}[\mathrm{Al}(\mathrm{OH})]^{2+} / \mathrm{HY}$

(h)

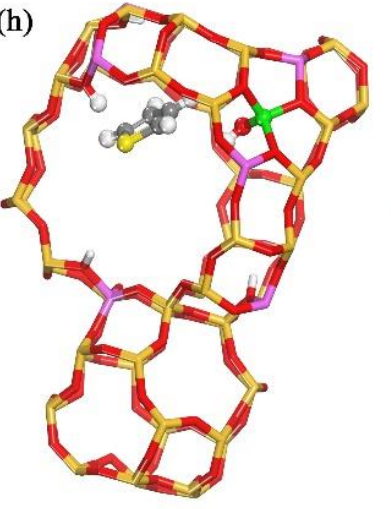

$\mathrm{C}_{4} \mathrm{H}_{4} \mathrm{~S}-\mathrm{NA}-[\mathrm{Al}(\mathrm{OH})]^{2+} / \mathrm{HY}$

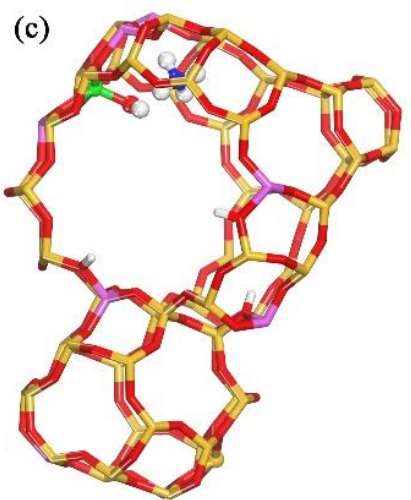

$\mathrm{NH}_{3}-\mathrm{A}-[\mathrm{Al}(\mathrm{OH})]^{2+} / \mathrm{HY}$

(f)

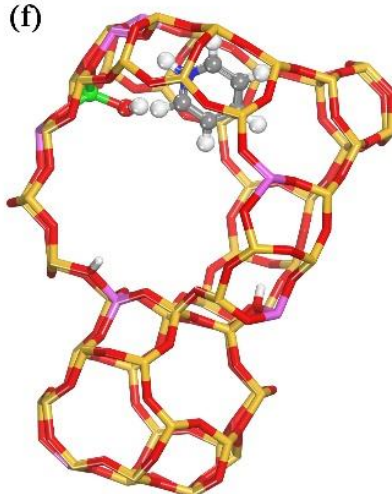

$\mathrm{C}_{5} \mathrm{H}_{5} \mathrm{~N}-\mathrm{A}-[\mathrm{Al}(\mathrm{OH})]^{2+} / \mathrm{HY}$

(i)

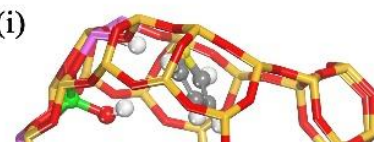

$\mathrm{Si}$

(2) $\mathrm{Al}_{\mathrm{EF}}$

(2) $\mathrm{Al}_{\mathrm{F}}$

20

$\mathrm{H}$

C $\mathrm{C}$

$\mathrm{s}$

$\therefore \mathrm{N}$

Figure S2 (a)-(c) Optimized adsorption structures of ammonia, (d)-(e) pyridine, and (f)-(h) thiophene in $(\mathrm{a}, \mathrm{d}, \mathrm{g}) \mathrm{HY}$ and $(\mathrm{b}, \mathrm{e}, \mathrm{h}) \mathrm{NA}-[\mathrm{Al}(\mathrm{OH})]^{2+} / \mathrm{HY}$, and $(\mathrm{c}, \mathrm{f}, \mathrm{i}) \mathrm{A}-$ $[\mathrm{Al}(\mathrm{OH})]^{2+} / \mathrm{HY}$ models. 
Table S1 The change of the Mulliken charge of ammonia and each atom

\begin{tabular}{cccc}
\hline & $\mathrm{HY}$ & $\mathrm{NA}-[\mathrm{Al}(\mathrm{OH})]^{2+} / \mathrm{HY}$ & $\mathrm{A}-[\mathrm{Al}(\mathrm{OH})]^{2+} / \mathrm{HY}$ \\
\hline$\Delta q(\mathrm{~N})$ & 0.089 & 0.098 & 0.072 \\
$\Delta q(\mathrm{H} 1)$ & 0.203 & 0.197 & 0.121 \\
$\Delta q(\mathrm{H} 2)$ & 0.109 & 0.116 & 0.168 \\
$\Delta q(\mathrm{H} 3)$ & 0.1 & 0.106 & 0.112 \\
$\Delta \mathrm{q}\left(\mathrm{H}^{+}\right)$ & -0.015 & -0.017 & 0.008 \\
$\Delta q\left(\mathrm{NH}_{3}\right)$ & 0.501 & 0.517 & 0.473 \\
$\Delta q\left(\mathrm{NH}_{4}^{+}\right)$ & 0.486 & 0.500 & 0.481 \\
\hline
\end{tabular}


Table S2 The change of the Mulliken charge of pyridine and each atom

\begin{tabular}{|c|c|c|c|}
\hline & HY & $\mathrm{NA}-[\mathrm{Al}(\mathrm{OH})]^{2+} / \mathrm{HY}$ & $\mathrm{A}-[\mathrm{Al}(\mathrm{OH})]^{2+} / \mathrm{HY}$ \\
\hline$\Delta q(\mathrm{~N})$ & 0.046 & 0.043 & 0.068 \\
\hline$\Delta q(\mathrm{C} 1)$ & -0.003 & 0.008 & 0.030 \\
\hline$\Delta q(\mathrm{C} 2)$ & -0.050 & -0.068 & -0.017 \\
\hline$\Delta q(\mathrm{C} 3)$ & -0.011 & -0.022 & -0.009 \\
\hline$\Delta q(\mathrm{C} 4)$ & -0.038 & -0.039 & -0.027 \\
\hline$\Delta q(\mathrm{C} 5)$ & 0.013 & 0.006 & 0.020 \\
\hline$\Delta q(\mathrm{H} 1)$ & 0.169 & 0.138 & 0.087 \\
\hline$\Delta q(\mathrm{H} 2)$ & 0.108 & 0.147 & 0.099 \\
\hline$\Delta q(\mathrm{H} 3)$ & 0.07 & 0.07 & 0.083 \\
\hline$\Delta q(\mathrm{H} 4)$ & 0.082 & 0.078 & 0.088 \\
\hline$\Delta q(\mathrm{H} 5)$ & 0.115 & 0.120 & 0.120 \\
\hline$\Delta q\left(\mathrm{H}^{+}\right)$ & 0.027 & 0.022 & 0.001 \\
\hline$\Delta q(\mathrm{C} 5 \mathrm{H} 5 \mathrm{~N})$ & 0.501 & 0.495 & 0.542 \\
\hline$\Delta q\left(\mathrm{C} 5 \mathrm{H} 5 \mathrm{~N}^{+}\right)$ & 0.528 & 0.517 & 0.543 \\
\hline
\end{tabular}


Table S3 The change of the Mulliken charge of thiophene and each atom

\begin{tabular}{cccc}
\hline & $\mathrm{HY}$ & $\mathrm{NA}-[\mathrm{Al}(\mathrm{OH})]^{2+} / \mathrm{HY}$ & $\mathrm{A}-[\mathrm{Al}(\mathrm{OH})]^{2+} / \mathrm{HY}$ \\
\hline$\Delta q(\mathrm{~S})$ & -0.115 & -0.102 & -0.101 \\
$\Delta q(\mathrm{C} 1)$ & -0.000 & -0.022 & 0.02 \\
$\Delta q(\mathrm{C} 2)$ & -0.015 & -0.019 & -0.001 \\
$\Delta q(\mathrm{C} 3)$ & -0.009 & 0.003 & -0.018 \\
$\Delta q(\mathrm{C} 4)$ & -0.013 & -0.002 & -0.035 \\
$\Delta q(\mathrm{H} 1)$ & 0.106 & 0.089 & 0.085 \\
$\Delta q(\mathrm{H} 2)$ & 0.026 & 0.059 & 0.027 \\
$\Delta q(\mathrm{H} 3)$ & 0.031 & 0.021 & 0.029 \\
$\Delta q(\mathrm{H} 4)$ & 0.072 & 0.043 & 0.101 \\
$\Delta q(\mathrm{C} 4 \mathrm{H} 4 \mathrm{~S})$ & 0.083 & 0.070 & 0.107 \\
\hline
\end{tabular}

Table S4 The change of the Mulliken charge of the $\mathrm{O}$ and $\mathrm{H}$ atoms of $[\mathrm{Al}(\mathrm{OH})]^{2+}$ species

\begin{tabular}{cccc}
\hline EFAl & $\mathrm{NH}_{3}$ & $\mathrm{C}_{5} \mathrm{~N}_{5} \mathrm{~N}$ & $\mathrm{C}_{4} \mathrm{~N}_{4} \mathrm{~S}$ \\
\hline $\mathrm{O} /[\mathrm{Al}(\mathrm{OH})]^{2+}$ & -0.015 & -0.054 & -0.016 \\
$\mathrm{H} /[\mathrm{Al}(\mathrm{OH})]^{2+}$ & -0.004 & 0.017 & 0.013 \\
\hline
\end{tabular}

